\title{
Technical Review on WSN Monitored High End Data Acquisition System using IEEE802.15.4 Wireless Protocol
}

\author{
Priyanka A. Nikhate ${ }^{1}$, C. Satyanarayana ${ }^{2}$ \\ ${ }^{I}$ Dept. of Electronics and Communication Engineering, Ballarpur Institute of Technology, Bamni, Ballarpur \\ (M.S.), India \\ ${ }^{2}$ Dept. of Electronics and Communication Engineering, Assistant Professor, Ballarpur Institute of Technology, \\ Bamni, Ballarpur (M.S.), India
}

\begin{abstract}
In this paper we describe the development of IEEE 802.15.4 compatible wireless sensor network for data acquisition with timing and without timing. We analyze the power consumption with timing and without timing. Data acquisition system playing on influencing role for monitoring and mentoring application for industrial culture. Sensor and I/O calibration on technical for PLC's highly demands the data base managing for research development and quality up graduation processes. Number of sensors are analog sensors few are dependent on digital or hybrid module. Many times need comes to transmit their responses \& acknowledgment over long distance \& store that in some software ERP systems. Traditionally wired system with or without current purposes. Our project research work briefly focuses on solution to overcome this difficulties in data acquisition with improved noise immunity cost redundancy \& guaranteed \& quality signal transmission and receiving outcomes. Since such system demand wireless technology with low transmission rates \& moderate distances for communication, zigbee will be highly demanded air interface for this. Due to inherent zigbee properties which are standardize with IEEE802.15.4. Zigbee will provide robust, strong, secular \& low cost application.
\end{abstract}

Keywords: Wireless sensor network, Network topology, Zigbee.

\section{Introduction}

Wireless technology, which has boomed in the IT sector over the past year, can be suitable for industrial control network as well, providing solution with high ROI with diagnostic, control and safety. In managing the move to wireless, it is clear that the common wireless protocol such as Wi-Fi, Bluetooth can be utilized on the factory floor. The challenge is to understand how to utilized wireless solution developed for IT applications as replacement for wired system in time critical scenarios typical of factory floor domain.

To date, most wireless system in production system are focused on application that require polling frequencies on the order of seconds or longer. However, the fundamental capabilities of these protocols allow support of much higher speed application such as motion control and close loop distributed logic. In past tends the star topology is used due to this the limited sensors are attached in node. But advancement in future is mesh topology is used for data acquisition, in mesh topology multiple sensors are used in multiple nodes. We analyze the power consumption due to giving timing interval, wireless data with slow compression and fast compression, wireless data with slow open and fast open with varying temperature.

\section{Litrature Review}

David Macarena's, Eric Flynn and Michael Todd [1] Wireless Sensor Network (WSN) for structural health monitoring (SHM) application can allow for a rapid assessment of structural integrity after an event such as natural disaster puts the reliability of civil infrastructure in question. Unfortunately, there are many technical challenges associated with employing such a WSN in civil infrastructure for operation over multiple decades. Maintenance cost must remains low enough to justify the integration of such a WSN in a given structure. The technical challenges include insuring power is delivered to the sensor node, reducing installation and maintenance costs and automating the collection and analysis of data collected by a wireless sensor network. Here we explore possible solutions to the technical challenges presented by WSN for SHM application. A "mobile host" WSN has been developed where a civil structure is instrumented with sensor nodes capable of being powered solely on energy transmitted to the sensor node wirelessly by the mobile host. When the sensor node has received adequate energy for making a given measurement, the sensor node performs the necessary measurement operation and then wirelessly transmits the measurement to the mobile host. These operations are then repeated for all desired sensor nodes in the network.

Brain Park Simeon Realov [2] we are proposing to design and implement a solar-powered wireless network for remote sensory data acquisition. With data being transmitted wirelessly over distances of up to 1.6 $\mathrm{km}$ and power harvested form solar energy, such a network would be ideally suited for situations in which 
sensory data needs to be gathered from remote open locations with no access to ground power. In order to ensure minimum power consumption, all of the digital circuitry is going to be powered by $3.3 \mathrm{~V}$ and the wireless transreceivers used are going to conform to the low power zigbee standard (IEEE 802.15.4). Constant power input will be maintained using a simple trickle charge connected to a rechargeable battery.

Li Cheng (2014) [3] Wireless sensor network node includes: the perception component, embedded processor, memory, and communication software components this several parts. Wireless sensor network is composed of one or more base stations (Sink node) and a large number of deployed in the monitoring area. This paper proposes design and implementation of monitoring information system based on wireless sensor network technology. The monitoring system is composed of sensor network terminal node, a number of small amounts of the sink node, a gateway node and the background monitoring system.

Stefan Koss, Helmut Dispert and Thorsten Knutz [4] In this paper we describe the devlopment of an IEEE 802.15.4 compatible wireless sensor network(WSN) node. The sensor node will acquire and internally store data periodically. Starting times as well as the time intervals for measurements can be freely programmed over the network system. As soon as a mobile network is detected in its proximity the node will automatically transfer data. Optionally sensor data can be delivered on demand. When in its idle state the node remains in power-down mode in order to minimize power consumption.

Gregory Mitchell, Marvin Conn, Russell Harris, and Andrew Bayba (July 2008) [5] The military has mandated the need for continuous health monitoring to be implemented for prognostics and Diagnostics (PD) applications at the mechanical and electronic level of fielded systems. The varity of military systems dictates the need for a highly flexible design that can be easily modified for different applications. Data acquisition is a core component of the PD process to get valuable information from the monitored system to a processing module for the execution of PD algorithums.

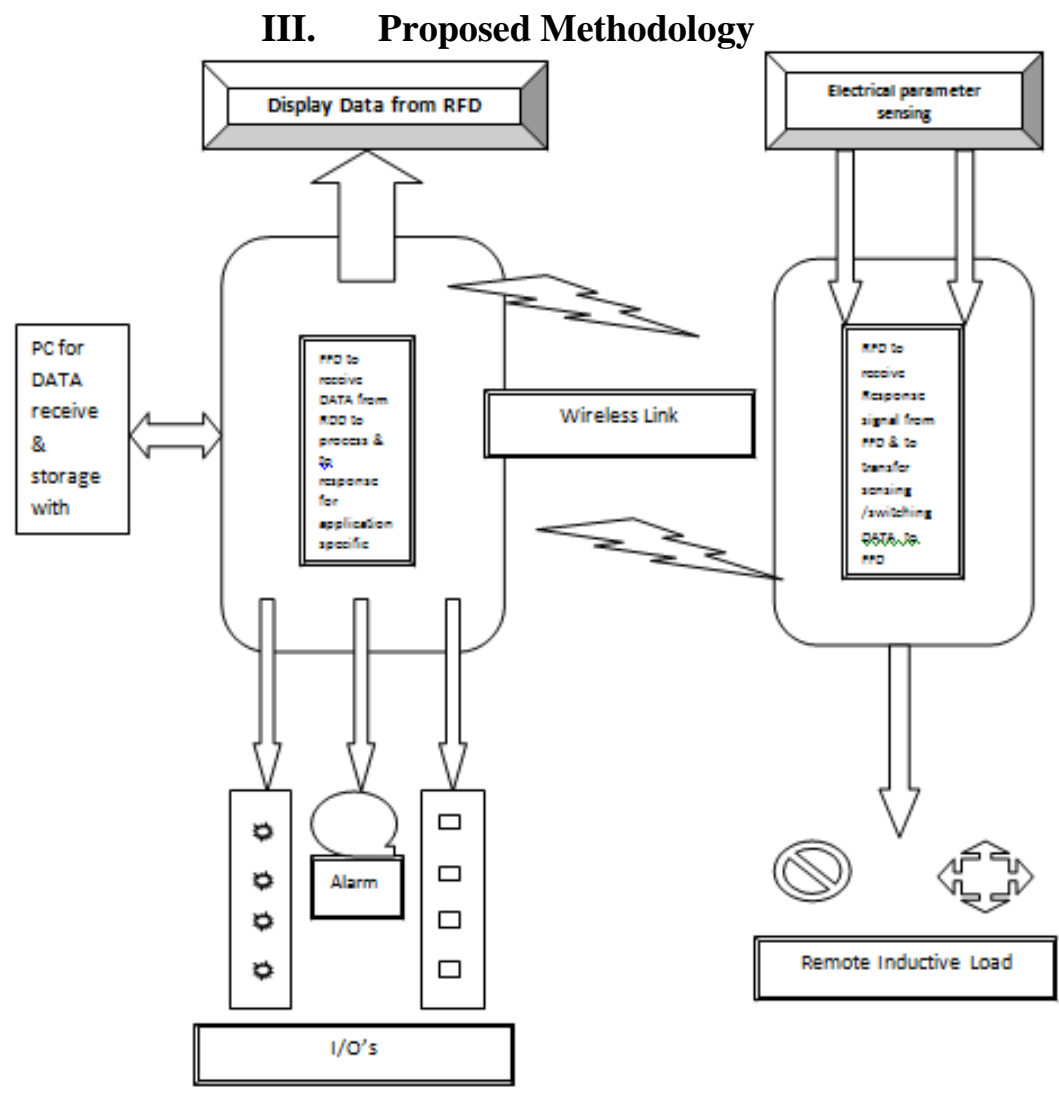

The project research work keep the model in which remote PLC control industrial machineries will be controlled \& regaled by the switching actions performed by FFD side. This FFD side also displays the status of the RFD side system on the LCD connected on its panel. Alarm \& Indications shown FFD side also displays the status of the RFD side system on the LCD connected on its panel. Alarm \& Indicators as shown that corresponds to status monitoring effects on server side due to remote machine parameter dependency.

As shown RFD side will be switched by FFD for production controlled mechanisms. Secondly electrical parameters will also be sensed on this for the purpose of data transmission to FFD. Both the devices 
Technical Review on WSN Monitored High End Data Acquisition System using IEEE802.15.4....

will be wirelessly linked by Zigbee with STAR topology. Industrial Automation profile of the Zigbee will be implemented here.

\subsection{Microcontroller \& Zigbee Module}

In the application development, microcontroller mainly meant for the function of the data processing, data storage, human interfacing and interoperability with the external environment. While developing any microcontroller the things to that can be taken into account are:

a) To choose requirement specific microcontroller with further scope of up gradation.

b) To study development tools with their version specification.

c) To study and apply High Level Language specification for application development as per complier variations.

The basic architectural \& peripheral requirement for microcontroller are:-

Instruction and data are on the separate buses, increasing speed \& overall performance.

a) While instruction fetching on program bus, data can be read or written on data bus.

b) Program Memory Bus optimized to any widths that fit within the design goals of microcontroller.

c) Wider buses which may allow more information to be transferred from memory to the CPU, enabling many instructions to be executed in a single cycle.

d) Instruction are upward compatible to maximize processing efficiency.

For our project work we are going for the controllers and developments tools of Microchip Technology. Module of CC2500 will be used here as a part of zigbee stack which will then be addressed by mixed API mode with hardware controlled flow control for USART interfacing with the desired baud rate.

IV. Result

\begin{tabular}{|c|c|c|c|c|c|c|c|c|c|c|c|c|c|c|c|}
\hline \multicolumn{10}{|c|}{ WSO } & \multicolumn{10}{|c|}{27} & \\
\hline Temp & 16 & 18 & 20 & 22 & 24 & 25 & 27 & 29 & 31 & 33 & 34 & 36 & 38 & 40 & 42 \\
\hline X & 273 & 273 & 273 & 273 & 273 & 273 & 273 & 273 & 273 & 273 & 273 & 273 & 273 & 273 & 273 \\
\hline Y & 511 & 511 & 511 & 511 & 511 & 511 & 511 & 511 & 511 & 511 & 511 & 511 & 511 & 511 & 511 \\
\hline Z & 464 & 464 & 464 & 464 & 464 & 464 & 464 & 464 & 464 & 464 & 464 & 464 & 464 & 464 & 464 \\
\hline Power & 1.35 & 1.49 & 1.65 & 1.82 & 2 & 2.12 & 2.25 & 2.46 & 2.62 & 2.79 & 2.87 & 3.00 & 3.17 & 3.33 & 3.52 \\
\hline
\end{tabular}

\begin{tabular}{|c|c|c|c|c|c|c|c|c|c|c|c|c|c|c|c|}
\hline \multicolumn{10}{|c|}{ WFO } \\
\hline Temp & 16 & 18 & 20 & 22 & 24 & 25 & 27 & 29 & 31 & 33 & 34 & 36 & 38 & 40 & 42 \\
\hline $\mathrm{X}$ & 273 & 273 & 273 & 273 & 273 & 273 & 273 & 273 & 273 & 273 & 273 & 273 & 273 & 273 & 273 \\
\hline $\mathrm{Y}$ & 511 & 511 & 511 & 511 & 511 & 511 & 511 & 511 & 511 & 511 & 511 & 511 & 511 & 511 & 511 \\
\hline $\mathrm{Z}$ & 464 & 464 & 464 & 464 & 464 & 464 & 464 & 464 & 464 & 464 & 464 & 464 & 464 & 464 & 464 \\
\hline Power & 1.33 & 1.55 & 1.7 & 1.83 & 2.03 & 2.08 & 2.29 & 2.43 & 2.65 & 2.83 & 2.88 & 3.03 & 3.19 & 3.33 & 3.52 \\
\hline
\end{tabular}

\begin{tabular}{|c|c|c|c|c|c|c|c|c|c|c|c|c|c|c|c|}
\hline \multicolumn{10}{|c|}{ WSC } \\
\hline Temp & 16 & 18 & 20 & 22 & 24 & 25 & 27 & 29 & 31 & 33 & 34 & 36 & 38 & 40 & 42 \\
\hline $\mathrm{X}$ & 273 & 273 & 273 & 273 & 273 & 273 & 273 & 273 & 273 & 273 & 273 & 273 & 273 & 273 & 273 \\
\hline $\mathrm{Y}$ & 511 & 511 & 511 & 511 & 511 & 511 & 511 & 511 & 511 & 511 & 511 & 511 & 511 & 511 & 511 \\
\hline $\mathrm{Z}$ & 464 & 464 & 464 & 464 & 464 & 464 & 464 & 464 & 464 & 464 & 464 & 464 & 464 & 464 & 464 \\
\hline Power & 0.33 & 0.41 & 0.68 & 0.87 & 1 & 1.12 & 1.25 & 1.55 & 1.64 & 1.79 & 1.97 & 2.24 & 2.34 & 2.46 & 2.61 \\
\hline
\end{tabular}

\begin{tabular}{|c|c|c|c|c|c|c|c|c|c|c|c|c|c|c|c|}
\hline \multicolumn{10}{|c|}{ WFC } \\
\hline Temp & 16 & 18 & 20 & 22 & 24 & 25 & 27 & 29 & 31 & 33 & 34 & 36 & 38 & 40 & 42 \\
\hline $\mathrm{X}$ & 273 & 273 & 273 & 273 & 273 & 273 & 273 & 273 & 273 & 273 & 273 & 273 & 273 & 273 & 273 \\
\hline $\mathrm{Y}$ & 511 & 511 & 511 & 511 & 511 & 511 & 511 & 511 & 511 & 511 & 511 & 511 & 511 & 511 & 511 \\
\hline $\mathrm{Z}$ & 464 & 464 & 464 & 464 & 464 & 464 & 464 & 464 & 464 & 464 & 464 & 464 & 464 & 464 & 464 \\
\hline Power & 0.38 & 0.44 & 0.72 & 0.89 & 1.03 & 1.16 & 1.29 & 1.57 & 1.61 & 1.83 & 2.07 & 2.28 & 2.38 & 2.51 & 2.6 \\
\hline
\end{tabular}



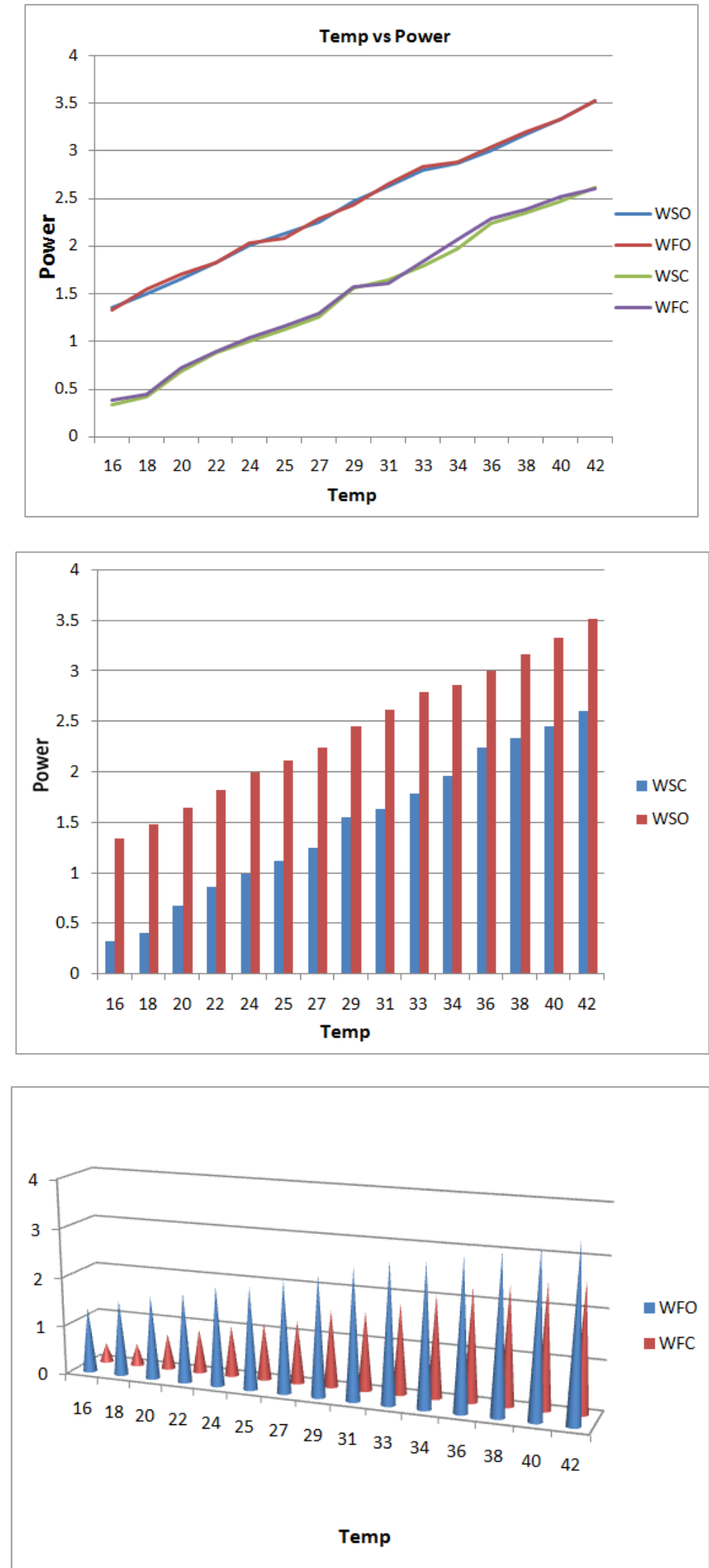

\section{Conclusion}


1. In this project, we have tried to determine performance of wireless technology in data acquisition with low power consumption and low cost. By using graphs, power consumption in WSC and WFC mode is less than WSO and WFO mode.

2. We are using three sensors which is $\mathrm{X}, \mathrm{Y}, \mathrm{Z}$.

3. We compress the data with $28 \%$.

4. When button 1 is press we send the data fast and slow.

5. When button 2 is press we send the data compression and expansion.

6. In wireless data with slow compress the power consumption is slowly increase than other.

7. In wireless data with fast compress the power consumption is fast increase.

8. Temperature sensor deep in water then they show the temperature of water.

9. In wireless data with slow open the power consumption is slowly increase.

10. In wireless data with fast open the power consumption is fast increase.

\section{References}

[1] David Mascarenas, Eric Flynn and Michael Todd "Wireless Sensor Technologies for Monitoring Civil Structure" University of California San Diego, California Gyuhae Farrar, Los Alamos National Laboratory, Los Alamos, New Mexico.

[2] Brian Park Simeon Realov "A Solar-Powered Wireless Data Acquisition Network"

[3] Li Cheng "Design and implementation of monitoring information system based on wireless sensor network technology.' Journal of Chemical and Pharmaceutical Reserch,2014,6(7):1685-1688.

[4] Stefan Koss, Helmut Dispert and Thorsten Knutz "Development of Zigbee-based wireless sensor network node for automatic data acquisition and transfer"

[5] Gregory Mitchell,Marvin Conn,Russell Harris, and Andrew Bayba "Automated Data Acquisition for a Prognostics and Diagnostics Health Monitoring System” Army Research Laboratory Adelphi,MD 20783-1197,July 2008.

[6] LAN-MAN Standard Committee of the IEEE Computer Society, Wireless Medium Access Personal Area, Networks (LR-WPANs), IEEE, 2003.

[7] Institute of Electrical and Electronics Engineers. IEEE std 802.11-2007, Wireless LAN Medium Access Control (MAC) and Physical Layer (PHY) specifications, 12 June 2007.

[8] ZIGBEE HOME AUTOMATION PUBLIC APPLICATION PROFILE from Zigbee Standards Organization Zigbee Profile:0x0104, Revision 25, Ver: 1.0.

[9] A Zigbee-based network for Home Control by Mario Collotta, Giuseppina Nicolosi, Emanuele Toscano, Orazio Mirabella. 